\title{
MARCADORES DISCURSIVOS EN NIÑOS DE 7 AÑOS CON TRASTORNO ESPECÍFICO DEL LENGUAJE: ESTUDIO DESCRIPTIVO ${ }^{1}$
}

\author{
DISCURSIVE MARKERS IN 7-YEAR-OLD CHILDREN WITH \\ SPECIFIC LANGUAGE IMPAIRMENT: DESCRIPTIVE STUDY
}

\author{
SARA ASENJO \\ Pontificia Universidad Católica de Valparaíso \\ sara.asenjo.s@mail.pucv.cl \\ ROGELIO NAZAR \\ Pontificia Universidad Católica de Valparaíso \\ rogelio.nazar@pucv.cl
}

\section{RESUMEN}

Esta investigación describe el uso de marcadores discursivos en 25 niños de 7 años con trastorno específico del lenguaje (TEL) en contraste con un grupo control de igual número y edad con desarrollo típico (DT). La motivación para el estudio se funda en que la cohesión ha sido indicada como un elemento de dificultad en niños con TEL, lo que puede ser perjudicial para su futuro desempeño académico y en su vida cotidiana (Soriano y Contreras, 2012; Buiza, Rodríguez y Adrián, 2015; Pérez, 1997; Acosta, Hernández y Ramírez, 2018). Para ampliar los resultados con respecto a estudios anteriores, la presente investigación combina perspectivas cuantitativa y cualitativa en el análisis de un corpus compuesto por una tarea de recontado oral con el apoyo visual de un libro álbum. Se analiza la cantidad de conectores por texto, por categoría y las reproducciones de conectores presentes en el texto original, comparando resultados de sujetos con TEL y DT. Se observa que no existen diferencias significativas entre los dos grupos muestrales en cuanto al uso de conectores. Solo se destaca un mayor uso de conectores coordinantes y un menor nivel de fidelidad con respecto al texto que se está recontando en los niños con TEL, lo cual se corresponde con características del trastorno.

Palabras clave: Cohesión, marcadores discursivos, tarea de recontado, trastorno específico del lenguaje.

${ }^{1}$ Esta investigación se circunscribe en el marco del Proyecto de investigación Fondecyt Regular 1191481, "Inducción automática de taxonomías de marcadores discursivos a partir de corpus multilingües", dirigido por Rogelio Nazar. 


\begin{abstract}
In this research we describe the use of discourse markers in 25 seven-year-old children diagnosed with specific language impairment (SLI) in contrast with a control group consisting of the same number of typically developing (TD) children of the same age. The motivation is grounded in that cohesion has been indicated as one of the challenging elements for children with this diagnosis, and problems with cohesion can be detrimental for their future academic performance and daily life (Soriano y Contreras, 2012; Buiza, Rodríguez y Adrián, 2015; Pérez, 1997; Acosta, Hernández y Ramírez, 2018). In order to expand the results of previous studies, in the present research we combine quantitative and qualitative perspectives to study the use of discourse markers in a corpus elicited by a story retelling task using a picture book. We analyze the number of discourse markers per text as well as their categories and their coincidence with the markers used in the original story, comparing the results of SLI and TD subjects. We observe no significant difference between the two groups regarding the use of discourse markers. There is only a slightly greater use of coordinating connectors and less coincidence with the markers used in the original story in the case of the SLI group, which is consistent with characteristics of this diagnosis.
\end{abstract}

Keywords: Cohesion, discourse markers, specific language impairment, story retelling task.

Recibido: 01/10/2019. Aceptado:15/01/2020.

\title{
1. INTRODUCCIÓN
}

T as personas con trastorno específico del lenguaje (TEL) poseen dificultades en el desarrollo de sus habilidades lingüísticas en distintos niveles, ya sea fonológico, morfológico, sintáctico, e incluso pragmático, en comparación con niños con desarrollo típico (DT). Específicamente en el ámbito discursivo y desde la dimensión microtextual del lenguaje, tener dificultades en la conexión de frases ha sido indicado como un aspecto característico del trastorno según el DSM-V (Asociación Americana de Psiquiatría, 2014). Desde esta perspectiva, la cohesión es una propiedad del texto que permite la continuidad de sus elementos dotándolo de una estructura (Van Dijk, 1978; De Beaugrande y Dressler, 1981), lo cual es posible gracias al enlace de segmentos lingüísticos. Ante lo dicho, algunos autores plantean las deficiencias en la organización narrativa de los niños con este diagnóstico (Soriano y Contreras, 2012; Buiza et al., 2015). Se detectan problemas en los mecanismos de cohesión léxicos y gramaticales (Figueroa, Menares, Naranjo y Vergara, 2015) y más específicamente en la pronominalización y la elipsis (Pérez, 1997; Acosta, Hernández y Ramírez, 2018) o las relaciones causales y temporales (Coloma, 2014; Coloma, Mendoza y Carballo 2017). Sin embargo, tal como señalan Acosta et al. (2018), existen pocos estudios dedicados a los mecanismos de cohesión en personas con esta condición, a pesar de ser una de sus características. 
Una parte fundamental de los mecanismos de cohesión son los marcadores discursivos, definidos como elementos sintáctico-semánticos que toman distintas formas gramaticales (conjunciones, locuciones, adverbios, sintagmas verbales y preposicionales) y activan lazos preexistentes en el conocimiento de los interlocutores (Calsamiglia y Tusón, 1999; Martín Zorraquino y Portolés, 1999). Son elementos con contenido semántico procedimental y no léxico porque su contenido consiste en indicar al interlocutor cómo tiene que interpretar la secuencia de enunciados, con efectos cognitivos para la generación de inferencias (Blakemore, 2004). Los marcadores del discurso son, por tanto, esenciales para construir un texto comprensible, que tiene como eje guiar la interpretación del sujeto que recibe el mensaje para cumplir un objetivo específico. Algunos de estos elementos, además, tienen incidencia en la complejidad sintáctica, pues permiten la coordinación y subordinación de enunciados e implican el desarrollo de discursos más elaborados y complejos (Gili Gaya, 1980; Casado Velarde, 1993). Este punto ha sido reconocido como particularmente dificultoso en niños con TEL (Acosta, Ramírez y Hernández, 2018; Coloma, 2013; Alfaro, Crespo y Alvarado, 2016), puesto que es parte del desarrollo tardío del lenguaje y resulta importante como competencia lingüística que permite crear discursos requeridos en contextos académicos.

A pesar de su centralidad como mecanismos de cohesión, los estudios relativos a los marcadores discursivos en niños con TEL en lengua española no son numerosos, y menos lo son en las variantes latinoamericanas, con Jackson-Maldonado y Maldonado (2016) en México, y Crespo y Figueroa (2016) y Cisternas y León (2018) en Chile. Estos estudios indican que no hay diferencias significativas en la utilización de marcadores discursivos entre niños TEL y DT. Sin embargo, algunos de estos trabajos han abordado el fenómeno de forma general al tener como objetivo determinar un perfil narrativo de los TEL, teniendo el uso de los marcadores discursivos como uno entre distintos indicadores. Además, los estudios agrupan conjuntos de niños con diferentes edades, por lo cual se puede dificultar la comparación de esta característica en niños con DT. Por esta razón, el presente trabajo de investigación intenta describir específicamente los marcadores discursivos en un corpus constituido por narraciones de un grupo de 25 niños con TEL y 25 con DT, todos con edad de 7 años.

Los textos narrativos suelen ser utilizados para realizar estudios en TEL porque son el primer tipo textual en ser adquirido por los niños y resulta útil para observar el desarrollo lingüístico humano. Según Soriano y Contreras (2004), la elaboración de una narración conlleva pensar en las necesidades del receptor del mensaje y los objetivos comunicativos que implica. Por su parte, Adam y Lorda (1999) indican que este tipo textual plantea una secuencia de acciones motivadas y en un marco espacio-temporal específico, por lo cual su organización es esencial para que tenga una naturaleza narrativa como tal. En este tipo de texto, los marcadores discursivos posibilitan crear los vínculos que resultan en una estructura narrativa, 
ya que se requiere de diversos conectores temporales, espaciales y causales para una producción narrativa cohesiva.

En el análisis de esta investigación se utiliza la taxonomía de conectores de Calsamiglia y Tusón (1999) porque posee categorías propias de un texto narrativo y con usos que se ajustan a funciones básicas de relaciones semánticas. Otras taxonomías, como la de Martín Zorraquino y Portolés (1999), se preocupan de funciones más específicas, más propias de otros géneros como los argumentativos, y que son utilizados por oradores o escritores expertos. No serían tan adecuadas, por tanto, teniendo en cuenta que los autores de las narraciones aquí analizadas son sujetos de 7 años que todavía se encuentran en un nivel básico de dominio de la lengua.

En lo relativo al desarrollo típico, la etapa por la que atraviesan los niños de esta edad se conoce como el desarrollo tardío del lenguaje y tiene lugar entre los 3 y 6 años hasta la etapa de adolescencia (López-Ornat, 1999). Es en este momento en que las personas adoptan o desarrollan aún más el manejo de estructuras gramaticales complejas como la coordinación, la subordinación y los mecanismos de cohesión, y comienzan a su vez a elaborar discursos reales o ficticios. Marinkovich (1985) ya había señalado que los primeros conectores en adquirirse son las conjunciones y, porque y pero. Además, establece que el orden de adquisición de las relaciones semánticas es la adición, seguida de la causalidad y disyunción, para proseguir con la aparición de la temporalidad, adversatividad y consecutividad, siendo los marcadores de condicionalidad los últimos en ser producidos, entre los 3 y 4 años. En cuanto a las habilidades para narrar, es alrededor de los 4 años cuando se consigue la etapa de estructuración, con inicio, desarrollo y conclusión de lo contado (Maggiolo, Coloma y Pávez, 2009).

La cohesión realizada por medio de conectores en textos narrativos orales es por lo tanto importante a la edad de 7 años, en tanto es solicitado como objetivo de aprendizaje para que los estudiantes organicen sus ideas al narrar un texto, de acuerdo con el Programa de Lenguaje y Comunicación del Gobierno de Chile, desde 3ro básico (Mineduc, 2013). Por esta razón es útil conocer el estado del uso de este mecanismo de cohesión, como proyección a la trayectoria escolar de los niños y las posibles dificultades que podrían encontrar en su futuro. Naturalmente, esto permitiría determinar si es necesario potenciarlo mediante tratamientos o una educación adaptada a las necesidades de niños con TEL. En este sentido, es factible trabajar en personas con este diagnóstico y de esa edad, puesto que son parte del Programa de Integración Escolar (PIE), lo cual quiere decir que el sistema educativo se compromete a otorgar una educación adecuada para atender a las dificultades correspondientes al trastorno, con tratamiento hasta los 8 años.

Es por todo lo anteriormente mencionado que esta investigación tiene el objetivo de describir el uso de conectores en narraciones orales de niños de 7 años con TEL en comparación con niños con DT. Para ampliar la información aportada por 
investigaciones ya realizadas sobre el tema, se desarrolló el análisis de los marcadores discursivos de un corpus compuesto por narraciones a partir de una tarea de recontado. Estudiamos la cantidad de conectores a nivel general y por categoría, y medimos el grado de coincidencia entre los conectores presentes en el texto fuente y los que son reproducidos en los relatos de los niños. De esta manera, se trata de aportar información que pueda resultar de utilidad para crear tratamientos que fortalezcan el uso de este mecanismo de cohesión y apunten a una educación que responda a distintos tipos de aprendizaje, de modo que los niños con TEL tengan una mejor preparación para los desafíos de la vida académica y cotidiana.

\section{MARCO METODOLÓGICO}

Como ya se mencionó, este estudio tiene como objetivo describir el uso de conectores en narraciones orales de niños de 7 años con Trastorno Específico del Lenguaje en comparación con niños con Desarrollo Típico. La pregunta de esta investigación es si existen diferencias significativas en el uso de conectores entre ambos grupos.

\subsection{Corpus}

El corpus que es objeto del estudio proviene de una base de datos elaborada por el equipo dirigido por Nina Crespo en el contexto del Proyecto Fondecyt 1130420, "Factores lingüísticos y cognitivos que se relacionan con el desarrollo de la complejidad sintáctica oral en los primeros años escolares” (2013-2015). Está compuesto por narraciones producidas por un total de 50 niños de 7 años, escolarizados en instituciones de carácter público, particular-subvencionado y particular en Valparaíso, Viña del Mar y Concón. La participación de los sujetos quedó sujeta a la previa aceptación de un consentimiento informado y todo el proceso de recolección del corpus fue visado por el comité de ética de la Pontificia Universidad Católica de Valparaíso.

Del total de los textos, 25 fueron narrados por niños con un diagnóstico de TEL, quienes reciben tratamiento en escuelas de lenguaje y a los cuales se les aplicaron tres tests de selección: el Test exploratorio de gramática española A de Toronto (STSG-R) en su versión revisada por Pavez (2003), Test de Repetición de oraciones (STSG-E) y el Test para evaluar los procesos de simplificación fonológica en su versión revisada por Maggiolo et al. (2009). Los otros 25 textos forman parte de un grupo control y fueron narrados por sujetos con desarrollo típico, seleccionados de acuerdo con el criterio de sus profesores. 
En todos los casos, los textos se obtuvieron de una base de datos anonimizada, producidos mediante una tarea de recontado del cuento El Valiente Lilo, material inédito del Fondecyt 1130420 que reproducimos en el anexo con el permiso de las autoras. La tarea de recontado consiste en la reproducción oral de un texto que el emisor ha logrado dominar, lo cual se desarrolla a partir de tres pasos esenciales: en primer lugar, se visualiza el cuento en el formato de video; en segundo lugar, se revisa un libro con las imágenes que componen el cuento, sin ningún apoyo verbal y, por último, los niños reproducen el cuento de forma oral basándose en los elementos visuales del libro. Las narraciones fueron recolectadas por fonoaudiólogas especialmente instruidas para motivar la tarea de recontado. Las grabaciones fueron posteriormente transcritas fonética y ortográficamente en archivos de texto y segmentadas en cláusulas numeradas.

\subsection{Procedimientos de análisis}

Los pasos realizados para analizar el corpus se componen en primer lugar de la identificación de los conectores en los textos, seguida de su clasificación y cuantificación para su posterior comparación entre grupos. La clasificación de conectores se llevó a cabo a partir de las categorías planteadas por Calsamiglia y Tusón (1999), que consisten en las siguientes: aditivos, contrastivo/argumentativos, de base causal, temporales y espaciales. Esta taxonomía se utilizó para identificar conectores tanto en el texto base de la tarea de recontado como en el corpus producido por los niños con TEL y DT.

Se consideraron como aditivos aquellos conectores que añaden información que sigue una misma línea, como lo son $y$, además, encima, después, incluso, también, del mismo modo, etc, como se puede ver en el ejemplo (1).

\section{(1) Siguió los consejos que le dio su mamá y voló con mucha fuerza.}

En cuanto a los contrastivos o contraargumentativos, son los que cumplen la función de cambiar de manera parcial o total la orientación de una argumentación, como en el ejemplo (2). A esta categoría corresponden los conectores, pero, en cambio, sin embargo, ahora bien, sino, por el contrario y no obstante, entre otros. Junto con los aditivos, los contraargumentativos son conectores que permiten la coordinación de proposiciones.

\section{(2) Pensó que iba a volar, pero le dio mucho miedo.}

Los conectores de base causal son una macro categoría de la cual forman parte los causativos, consecutivos, condicionales y finales. Los causativos, tal como in- 
dica su nombre, establecen relaciones de causa entre las proposiciones, como en el ejemplo (3). Corresponden a esta categoría los conectores porque, por eso, pues, puesto que, entre otros. Los conectores consecutivos, en cambio, establecen relaciones de consecuencia con respecto a la proposición anterior, como en el ejemplo (4). Pertenecen a esta categoría conectores como entonces, asi que, pues, luego, etc. Así mismo, los conectores condicionales presentan la causa hipotética de una proposición ya presentada, como lo hacen si, cuando, mientras, a menos que, siempre que, como se ve en el ejemplo (5). La última categoría de conectores de base causal es la de los finales, que introducen la causa como una meta o propósito. Con este objeto se utilizan palabras como para que, con el propósito de que, a fin de que, cuyo ejemplo (6) de clasificación se presenta a continuación.

(3) Lilo pensaba que se iba a caer porque era un árbol muy alto.

(4) Se haría el enfermo, así que a la mañana siguiente dijo que le dolian los ojos.

(5) Si se hacia el enfermo, no tendría que volar

(6) Se hizo el enfermo para no ir.

Los conectores siguientes son los que permiten realizar una descripción del marco espacio-temporal de la narración, los conectores temporales y espaciales. Los primeros, representados en el ejemplo (7), permiten relacionar temporalmente dos proposiciones, como cuando, de pronto, entonces, luego, un dia, de repente. De la misma manera, los conectores espaciales permiten relacionar espacialmente dos proposiciones, como se puede apreciar en el ejemplo (8). Es el caso de enfrente, delante, detrás, al fondo, a la derecha, a la izquierda, etc.

\section{(7) Voló después de salvar a su hermana. \\ (8) Se puso al lado de él.}

Con base en esta taxonomía, se realizó de manera manual la detección y clasificación de los marcadores en todos los textos, incluyendo el texto fuente a partir de la transcripción del video que relata de forma oral la historia. Con el fin de evitar cualquier posibilidad de sesgo durante el análisis, los textos fueron anonimizados mediante un proceso automático (un script Perl que asigna identificadores numéricos a cada texto), de forma que resultara imposible si el texto que se está anotando pertenece a las producciones de TEL o DT. Para la identificación y clasificación de los conectores se ha procedido insertando corchetes para demarcar la ocurrencia de la unidad considerada como conector y, entre paréntesis e inmediatamente después, la clasificación correspondiente, como puede verse a continuación:

[póřke(causativo)] éra ún ářbol múi lárgo / 
Este procedimiento de marcado permitió llevar un registro de la frecuencia de los conectores y de sus categorías, como se presenta en el siguiente ejemplo:

Marcadores discursivos: 19

Aditivos: 8 (y)

Temporales: durante (2), después (3) $=5$

Contraargumentativo: 1 (pero)

Causativo: 3 (porque)

Consecutivo: 1 (entonces)

Final: 1 (para)

Del mismo modo llevamos también un registro de las coincidencias con los conectores utilizados en el texto fuente. Todos estos datos fueron a continuación organizados en una matriz en la que se dispone cada texto mediante su código de identificación, se descubre el diagnóstico correspondiente y la cantidad de conectores en total y por categoría.

A través de los procedimientos planteados, las variables investigadas fueron la cantidad de conectores totales por muestra, la cantidad de conectores por categoría y también la cantidad de reproducciones del texto fuente por grupo muestral. De acuerdo con estos datos se realizó estadísticas descriptivas utilizando el software R (R Foundation, 2020), generando diagramas de barras y diagramas de caja. Además, se aplicó una serie de t-tests para obtener el valor-p correspondiente a la probabilidad de una hipótesis nula según la cual no existirían diferencias significativas entre TEL y DT.

\section{RESULTADOS}

Los resultados que se describen a continuación corresponden a la cantidad de uso de conectores en total, la cantidad de conectores por categoría, la cantidad de reproducciones por texto y luego por categoría. En primer lugar, como resultado total del corpus se encontraron 1.079 conectores, de los cuales se muestra el porcentaje correspondiente a cada grupo muestral en el Gráfico 1.

Como se puede apreciar, el grupo con TEL tiene un menor uso de conectores a nivel general con respecto a los niños con DT, al que corresponde el 54\% de los casos de conectores de todo el corpus. Sin embargo, las diferencias con respecto a los elementos que fueron analizados no resultaron estadísticamente significativas según lo que arroja el t-test con un nivel alfa de 0,05, ni en total ni en cada una de las categorías por separado. En la Tabla I se puede observar el detalle de la estadística para ambos grupos, incluyendo el grado de reproducción de los conectores 
encontrados en la narración original. Se presenta la media y la desviación estándar por total, por categoría y por grupo, junto con el valor p que devuelve el t-test en cada comparación y el tamaño del efecto, utilizando la $d$ de Cohen. En ningún caso se aprecian valores p menores al alfa utilizado (aunque encontramos dos casos limítrofes que señalamos con asterisco) y en ningún caso el tamaño del efecto fue alto.

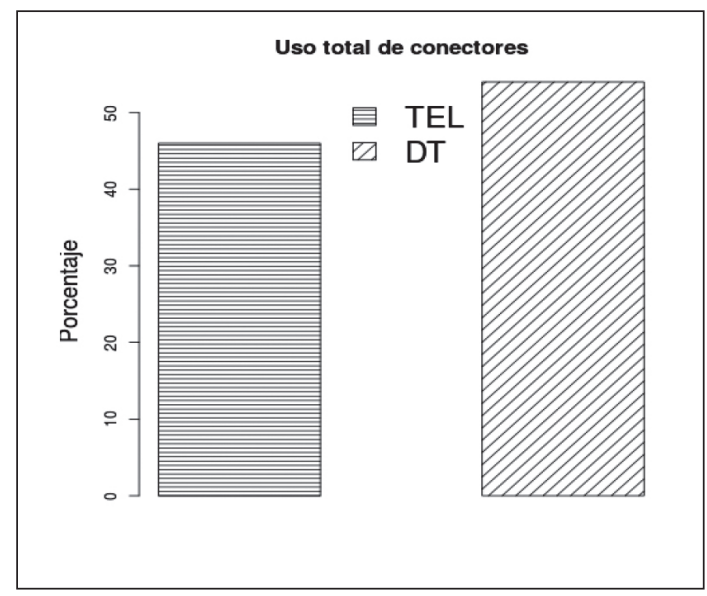

Gráfico 1. Porcentaje de conectores por grupo.

Tabla I. Resultados del análisis estadístico de los grupos TEL y DT (casos limítrofes del valor p marcados con ${ }^{*}$ ).

\begin{tabular}{|l|r|r|r|r|l|l|}
\hline Categoría & $\begin{array}{c}\text { Media } \\
\text { TEL }\end{array}$ & $\begin{array}{c}\text { Desv. est. } \\
\text { TEL }\end{array}$ & $\begin{array}{c}\text { Media } \\
\text { DT }\end{array}$ & $\begin{array}{c}\text { Desv. } \\
\text { est. DT }\end{array}$ & $\begin{array}{l}\text { Valor p } \\
\text { (t-test) }\end{array}$ & $\begin{array}{l}\text { Tamaño } \\
\text { del efecto } \\
\text { (d de Cohen) }\end{array}$ \\
\hline Total de conectores & 19,72 & 5,80 & 23,44 & 8,86 & 0,08 & $-0,50$ \\
\hline Aditivos & 10,72 & 4,60 & 11,68 & 4,50 & 0,46 & $-0,21$ \\
\hline Contraargumentativos & 1,24 & 1,39 & 0,88 & 0,97 & 0,29 & 0,30 \\
\hline Causativos & 1,4 & 1,15 & 2,28 & 2,40 & 0,10 & $-0,47$ \\
\hline Consecutivos & 0,96 & 1,74 & 1,36 & 1,89 & 0,44 & $-0,22$ \\
\hline Condicionales & 0,6 & 1 & 0,16 & 0,47 & $0,05^{*}$ & 0,56 \\
\hline Finales & 0,8 & 0,81 & 1,2 & 1,84 & 0,28 & $-0,28$ \\
\hline Temporales & 3,92 & 3,02 & 5,76 & 3,65 & $0,05^{*}$ & $-0,55$ \\
\hline Espaciales & 0,12 & 0,33 & 0,08 & 0,4 & 0,70 & 0,11 \\
\hline $\begin{array}{l}\text { Cantidad de conectores } \\
\text { reproducidos }\end{array}$ & 4,24 & 1,23 & 4,64 & 1,99 & 0,39 & $-0,24$ \\
\hline
\end{tabular}


Estos resultados muestran que no hay diferencias significativas en el uso de marcadores entre el grupo con TEL y el grupo control. De todas maneras, sí se aprecia que los conectores condicionales y temporales se acercaron al valor límite. Esto se puede apreciar mejor en el diagrama de barras del Gráfico 2, que exhibe la comparación del uso de conectores por categoría expresado en porcentaje.

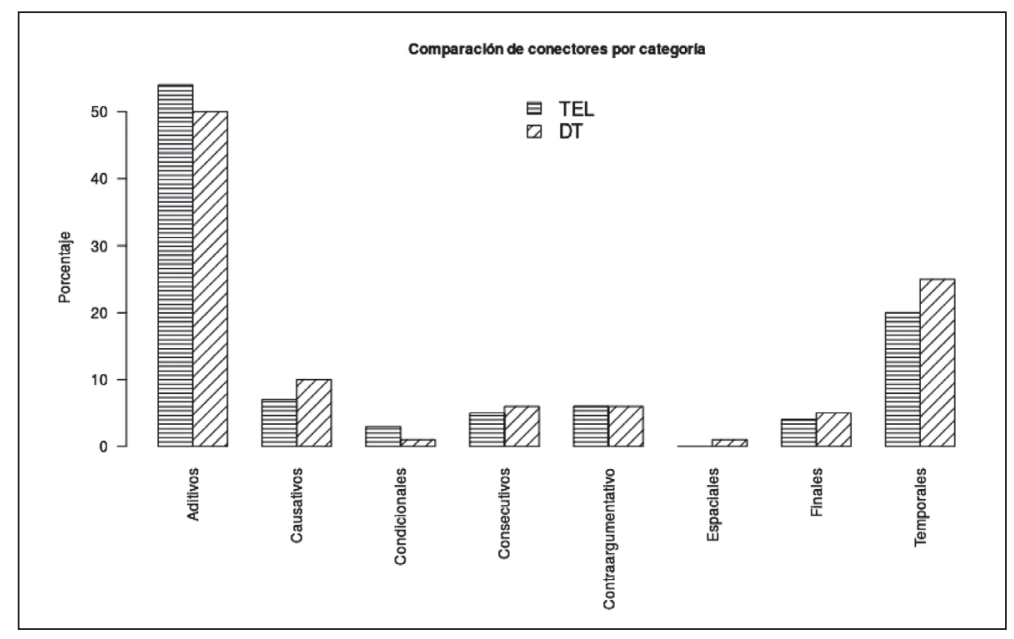

Gráfico 2. Gráfico de comparación de categorías por grupo.

A nivel general se puede observar que los conectores de mayor uso fueron los aditivos, seguidos de los temporales y los causativos para ambos grupos. En contraste, los conectores espaciales fueron los de menor uso, con solo 4 casos en los 50 textos analizados. A su vez, hay categorías en las cuales TEL presenta un mayor porcentaje con respecto a DT, como los aditivos, contraargumentativos, condicionales y espaciales. Por su parte, los niños con TEL presentan menor medida de categorías temporales, causativas, consecutivas y finales con respecto a DT. Estos resultados concuerdan con los conectores que se presentan en el texto original de El Valiente Lilo, tal como se puede observar en la Tabla II. 
Tabla II. Análisis de conectores en texto original de "El Valiente Lilo".

\begin{tabular}{|c|c|c|}
\hline Categoría & Casos & Conector utilizado \\
\hline Aditivos & 7 & $y$ \\
\hline Contraargumentativo & 2 & pero \\
\hline Causativo & 5 & $\begin{array}{l}\text { porque (3) } \\
\text { por }(1) \\
\text { pues }(1)\end{array}$ \\
\hline Consecutivo & 2 & $\begin{array}{l}\text { entonces } \\
\text { así }\end{array}$ \\
\hline Condicional & 1 & $s i$ \\
\hline Finales & 2 & $\begin{array}{l}\text { para que } \\
\text { para }\end{array}$ \\
\hline Temporales & 7 & $\begin{array}{l}\text { un dia }(1) \\
\text { mientras (3) } \\
\text { de repente (1) } \\
\text { a la mañana siguiente (1) } \\
\text { en ese momento (1) }\end{array}$ \\
\hline Espaciales & 1 & frente (1) \\
\hline Total & 27 & \\
\hline
\end{tabular}

$\mathrm{Al}$ igual que en los resultados de las producciones narrativas de los niños, en el cuento que les sirve de fuente los conectores de mayor uso son el aditivo $y$, luego los temporales y los causativos. En correspondencia con los conectores de menor uso, en el cuento solo se presenta un conector espacial y un conector condicional, lo cual lleva a suponer que al realizar una tarea de recontado los conectores del texto fuente afecten los resultados que presentan los niños en sus propias narraciones. De los conectores presentes en la Tabla II, la cantidad utilizada en las producciones de los niños se reflejan en el Gráfico 3. 


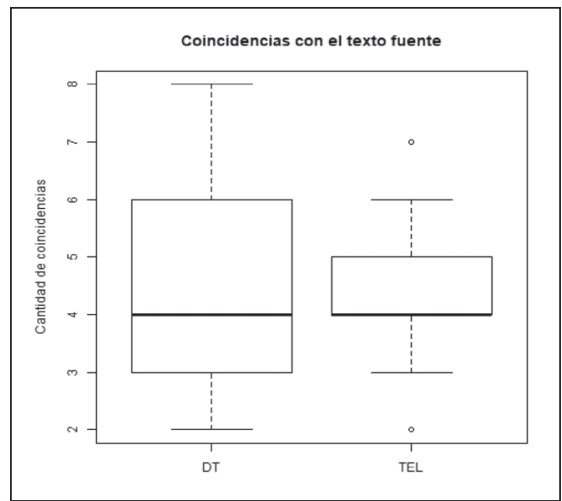

Gráfico 3. Diagrama de cajas de la cantidad de coincidencias con el texto original por población.

Los resultados en el diagrama de cajas permiten indicar que ambos grupos muestrales reproducen en promedio 4 de los conectores presentes en el texto fuente, pero TEL presenta menor variabilidad en los valores, pues DT concentra sus reproducciones de conectores entre 2 y 8 , lo cual es un espectro mayor que TEL, que concentra sus reproducciones entre 3 y 6 . Por ende, se puede señalar que el grado de dispersión es mayor en el grupo control que en el grupo con TEL, alcanzando valores más altos de reproducción de conectores. En este sentido, TEL es menos fiel al texto fuente que DT pero, aun así, existen algunas diferencias en lo relativo a la fidelidad del texto fuente en el uso por categoría, lo que se muestra en el Gráfico 4, en el cual se ha calculado el porcentaje por cantidad de textos que presenta el conector de la categoría correspondiente.

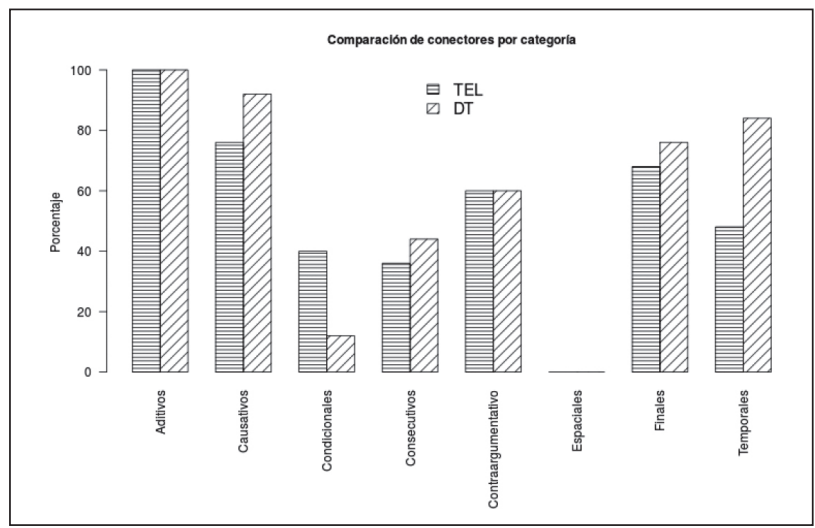

Gráfico 4. Gráfico de comparación de reproducciones del texto fuente entre DT y TEL. 
El gráfico muestra que el nivel de reproducción de conectores aditivos es de un $100 \%$ tanto en TEL como en DT. Así mismo, tienen exactamente el mismo porcentaje $(60 \%)$ en la categoría de contraargumentativos, ambas categorías correspondientes a la coordinación de enunciados. Para todas las otras categorías de conectores, TEL presenta un menor porcentaje con respecto a DT, excepto para el caso de los condicionales, cuya naturaleza es subordinante de enunciados. En concordancia con otros resultados ya presentados, los conectores que tienen mayor fidelidad de reproducción son los aditivos, los causativos, los finales en ambos grupos, y además los temporales en el caso de DT. Cabe destacar, también, que el conector espacial frente no tuvo ninguna reproducción por parte de ambos grupos, lo que quiere decir que los cuatro casos encontrados de conectores espaciales son todos parte del repertorio propio de los niños.

En suma, TEL tiene un menor uso de conectores con respecto a DT, si bien sus diferencias en el comportamiento de este elemento lingüístico no son lo suficientemente grandes como para ser estadísticamente significativas. En lo relativo a nivel de fidelidad del uso de conectores del texto fuente, el grupo con TEL se inclina en menor medida a los conectores presentes en el cuento reproducido. Además, se comprobó que los resultados de conectores por categoría se ven influenciados por aquellos utilizados en el texto fuente.

\section{DISCUSIÓN}

Los resultados explicitados por medio de los procedimientos de análisis ya mencionados han llevado a comprobar que, si bien existen diferencias en el uso de conectores, mostrando al grupo con DT con mayor uso, esta diferencia no resultó significativa. Esto coincidiría, en parte, con los resultados de Jackson-Maldonado y Maldonado (2016), quienes obtuvieron resultados similares. Ahora bien, cabe destacar que ellos hacen su análisis en el grupo total de niños de ambas poblaciones (7 con TEL y 9 con DT), cuyas edades fluctúan entre 5 y 9 años, sin distinguir dichos resultados por edades. En este sentido, la presente investigación logró identificar lo que ocurre en una edad determinada, cuya importancia radica en que se encuentran en la etapa escolar en que tendrán que avanzar en su aprendizaje para el incremento de su repertorio de conectores. Similares fueron los resultados de Coloma et al. (2017) con respecto a la cantidad de relaciones semánticas temporales y causales en una población de niños con TEL y DT, de 6,6 años en promedio, en el que se demostró que tampoco fueron significativas las diferencias. Los hallazgos de la presente investigación indican menor uso de conectores en el grupo TEL en comparación con el grupo DT, pero se puede señalar que los niños de ambos grupos se encuentran en un nivel de desarrollo de conectores similar 
cuando narran en forma oral. Sería ideal, sin embargo, contar con un tamaño muestral mayor para alcanzar resultados concluyentes.

Con respecto a las diferencias del uso de conectores en las distintas categorías, los resultados de la investigación se corresponden con los de otros estudios, en tanto el conector de mayor uso en ambos grupos es el aditivo coordinante $y$ (Kaderavek y Sulzby, 2000; Jackson-Maldonado y Maldonado, 2016; Cisternas y León, 2018). Esto se puede atribuir a que dicho conector es uno de los primeros en ser adquirido en el desarrollo temprano del niño (Bloom, Lahey, Lifter y Fiess, 1980; Marinkovich, 1985), por lo cual probablemente es aquel que se utiliza con mayor frecuencia en los textos narrativos. De la misma manera, este conector es uno de los que tiene mayor presencia en el texto base, lo cual concordaría con un uso predominante en términos de reproducción, con un nivel de fidelidad de $100 \%$ al realizar la tarea de recontado. Esta podría ser la razón por la cual los grupos de la muestra utilizan más este tipo de conector. En este sentido, también se puede plantear la poca variedad léxica y, por ende, un nivel cohesivo todavía básico, puesto que el uso de $y$ corresponde a más del 50\% de conectores presentes de todo el corpus (53\%). Si el conector aditivo $y$ es el primero en adquirirse, entonces los niños le atribuirían un uso multifuncional a nivel de relaciones semánticas entre proposiciones, aunque en edades más avanzadas irían logrando una mayor precisión a medida que se van dominando otros conectores (Marinkovich, 1985; Álvarez, 1996). Podría atribuirse a este motivo el que TEL presente un uso mayor de conectores aditivos que el grupo control, ya que indicaría que todavía no se han consolidado otras formas de relaciones semánticas que están presentes en el corpus.

Así mismo, al igual que en Coloma et al. (2017), no se evidenciaron diferencias significativas en las relaciones temporales ni en las causales, las cuales fueron las más utilizadas después del conector aditivo. Con respecto a los conectores temporales, esto indica que los niños con TEL y DT reconocen una progresión y secuencialidad de los eventos que les permite estructurar la narración, lo cual concuerda con Cisternas y León (2018), quienes llegan a las mismas conclusiones respecto a la estructuración narrativa de niños con TEL de 5 años. Por su parte, el que los causales sean la tercera categoría más utilizada por los grupos de la muestra se puede relacionar con los hallazgos de Crespo y Figueroa (2016), quienes identifican que tanto TEL como DT tienden a realizar una narrativa con una estructura basada en la causalidad. Así también, Gutiérrez-Clellen e Iglesias (1992) aseguran que durante los primeros años escolares los niños presentan una mayor coherencia causal en sus narraciones.

A pesar de estos hallazgos, es importante señalar que las categorías de base causal (causales, consecutivos, condicionales, finales) presentan un porcentaje mucho menor con respecto a las categorías más utilizadas en ambos grupos muestrales, lo que pareciera indicar que son relaciones semánticas que todavía se están desarro- 
llando. Este planteamiento coincide con el estudio de Acosta, González y Lorenzo (2011), en el cual los problemas con relaciones causales son uno de los aspectos lingüísticos que persistió después de una intervención a niños con TEL de 3 años, lo cual pareciera señalar que su desarrollo es más tardío y tiene una adquisición más lenta y compleja. Más específicamente, entre las categorías de base causal que son menores en TEL, se encuentran los consecutivos (entonces, asi que), lo cual podría tener relación con que estos conectores pueden introducir relaciones subordinadas, las cuales son parte del desarrollo tardío del lenguaje, constan de una mayor complejidad sintáctica y pueden ser un problema para el TEL, como lo plantean Acosta et al. (2018), Coloma (2013) y Alfaro et al. (2016).

Esto se condice con los resultados de reproducciones de conectores condicionales $(s i)$ y finales (para/para que) del texto base, los que no presentan variedad de conectores propios del repertorio de los niños y son de las categorías menos numerosas a nivel de porcentaje en el corpus de TEL y DT, indicando que son relaciones semánticas que todavía no se encuentran bien adquiridas en este momento del desarrollo de los niños. Otra de las razones que podrían explicar esta situación es justamente la pobreza de vocabulario, si bien no se encontraron registros cuantitativos de la variedad léxica de conectores específicamente. Buiza et al. (2015) plantean que existe dificultad de acceso al léxico en niños con TEL para tareas de definición y denominación y para buscar asociaciones semánticas en las palabras, lo cual sugiere una relación con restricciones cognitivas. En cuanto a esto, Aguado, Cuetos-Vega, Domezáin y Pascual (2006), Mantiñán, Badel y Fermoselle (2014,), Acosta, Ramírez y Hernández (2017) y Lepe (2018) indican que los niños con TEL tienen problemas en la memoria de trabajo verbal, principalmente en el almacén fonológico y con efectos en la repetición de palabras.

Relacionado con lo anterior, este antecedente coincide con los resultados generales de las reproducciones del texto fuente, pues los niños con TEL mostraron menor fidelidad al texto original en comparación con los DT en términos cuantitativos por categoría, si bien esta diferencia tampoco llega a ser estadísticamente significativa. En función de lo que implica cognitivamente la tarea de recontado, su demanda de atención y el importante uso de la memoria de trabajo, cabía esperar que, por sus características, a los niños con TEL les sea más dificultoso acceder a las palabras al momento de realizar la tarea, afectando entre ellas a los conectores que estaban presentes en el texto fuente. A ello se suma que en algunos casos los conectores están compuestos por preposiciones o sintagmas preposicionales, y en el caso específico del TEL, Crespo, Koza y Alfaro (2018) verificaron que en español los niños con este diagnóstico tienden a elidir o sustituir este tipo de palabras más que el grupo con DT, lo que podría haber afectado los resultados en la fidelidad de conectores. Sin embargo, la evidencia encontrada no es suficiente para afirmar esto con seguridad.

Otro hallazgo que llama la atención es la casi inexistente presencia de conecto- 
res espaciales. Esto es importante debido a que se trata de un texto narrativo en el cual es necesario insertar al individuo comprendedor en un marco espacial. Una interpretación podría ser que, dado que los niños que produjeron los textos tenían acceso a imágenes con representaciones del espacio en las que se podían explayar, dieron menor relevancia a este aspecto de la cohesión textual, lo cual tiene sentido bajo los planteamientos de Van Dijk (1978), quien señala que lo principal de una narración es la acción, a partir de lo cual la descripción de las circunstancias queda en un segundo plano. Además, esto podría tener relación con que los niños asumen que el interlocutor conoce el espacio, porque el espacio narrado se encuentra cercano al espacio de emisión, es decir, ellos tenían las imágenes en su mismo espacio de producción y todos los presentes las podían observar. Al respecto, Aravena (2010), en un estudio del desarrollo de las referencias de persona, tiempo y espacio, indica que a menor edad se suelen plantear locaciones espaciales menos elaboradas y que mientras más distante se encuentre el sujeto del marco espacial, hay mayor riqueza y complejidad en el uso de las locaciones. De todas maneras, la autora señala que, a nivel general en los distintos rangos etarios, las relaciones espaciales tienen una menor presencia con respecto a las otras referencias que ha estudiado, por lo cual esto no necesariamente tiene que ver con la edad o diagnóstico de los niños, sino con otros factores no considerados en esta investigación. Podría atribuirse a estos múltiples motivos el hecho de que los niños de la muestra no presentaran este tipo de marcadores, pero también podría ser que simplemente no consideraron necesario reforzar la dimensión espacial de la narración mediante conectores.

En definitiva, no observamos diferencias significativas en el uso de conectores entre los dos grupos muestrales. Las que se observan son leves, aunque sí se corresponden con la explicación que se da respecto a la adquisición y desarrollo lingüístico de los conectores en los niños, en el cual TEL está levemente desnivelado. Esto se produce, además, porque algunos conectores se relacionan con la complejidad en la elaboración del discurso, como los conectores que permiten la subordinación y que tienen una adquisición tardía. Otras diferencias, como la reproducción de conectores, podría estar relacionada con las características del trastorno, el cual presenta deficiencias en la memoria verbal, pero esto requeriría mayor investigación.

\section{CONCLUSIONES}

A través de los procedimientos de análisis para identificar y comparar la cantidad y variedad categorial de conectores producidos en los grupos muestrales, el estudio permitió verificar lo que ya habían evidenciado algunas investigaciones anteriores: que no hay diferencias significativas entre TEL y DT con respecto a su desempeño 
relativo a las variables antes señaladas. Esto quiere decir que el grupo TEL y DT presentan un uso de conectores no idéntico pero similar, según las diferencias que hemos encontrado y descrito. La investigación también aporta información con respecto a una edad específica y distinta a las ya abarcadas por otros estudios que abordan el mismo fenómeno en narraciones (Jackson-Maldonado y Maldonado, 2016; Coloma et al. 2017; Cisternas y León, 2018). Entre los hallazgos, los niños con TEL de 7 años, al igual que los DT de la misma edad, usan en mayor medida conectores coordinantes y en menor medida los subordinantes, lo que pareciera indicar una falta del desarrollo de nexos que posibiliten la construcción de estructuras más complejas. Esto, además, es esperable en el momento de desarrollo en el que se encuentran.

A su vez, como principales aportes del presente estudio, se ha logrado evidenciar lo que ocurre con los conectores que no han sido el objeto central de otros análisis, tal como los conectores condicionales, los que presentan un porcentaje ligeramente mayor en las narraciones orales de niños con TEL que en DT. Esto resulta inesperado en vista de que implica construcciones sintácticas más complejas. De la misma manera, se evidencia un bajo uso de conectores referentes a la descripción del espacio y un nivel de fidelidad con el texto original en términos de uso de conectores que es menor en TEL, información que también era escasa en investigaciones previas en este diagnóstico.

Como proyección, sería interesante comparar la cohesión discursiva por medio de conectores y otros mecanismos, como la sustitución léxica, cuyo estudio también es escaso. Otra posibilidad sería enfocar la investigación científica a los conectores espaciales u otras formas de referencia espacial en las narraciones orales, los que no se encontraron en el presente estudio, para identificar qué factor podría estar incidiendo o comprobar si en otros tipos de relatos se repite el fenómeno, como en las relaciones temporales y su posible conexión con dificultades del uso de preposiciones. Sumado a lo anterior, se podría comprobar, mediante la comparación del uso de conectores en actividades de escritura con los presentes en actividades discursivas orales, si sus desaciertos a nivel léxico persisten en sus dificultades para establecer cohesión en sus textos orales y escritos. Al respecto, cabe referirse a lo señalado por Kaderavek y Sulzby (2000), para quienes las dificultades cohesivas de niños con TEL se presentan tanto en el ámbito oral como escrito. Otras posibilidades de trabajo futuro podrían ser incorporar nuevas variables, como por ejemplo el análisis de la riqueza de vocabulario en general y no solo de marcadores. Variables de otro tipo que también podrían ser consideradas son las categorías de TEL expresivo y mixto, que en nuestro caso no fueron estudiadas debido a las características del corpus.

Entre las variables que podrían tenerse en cuenta en investigaciones futuras también destacamos la del nivel sociocultural de los sujetos, ya que cabe esperar que sea un factor en los resultados. Sería relevante tomar en cuenta esta variable 
debido a que los conectores comienzan a desarrollarse tempranamente durante la infancia. Como plantea Vygotski (1981), el pensamiento y otras funciones cognitivas se establecen en tanto el ser humano es un sujeto social que se desarrolla gracias a y dependiendo de los procesos de socialización que se den en su contexto. Sin lugar a duda, el factor social es igualmente importante cuando se trata del uso del lenguaje, con repercusiones en el vocabulario como señalan Rosemberg, Menti, Stein, Alam y Migdalek (2016), pues se ha indicado que los niños utilizan las palabras a las que se han visto expuestos durante su desarrollo, ante lo cual la interacción con los adultos que se encuentran en su medio es decisiva.

Todo lo anterior constituiría un aporte en posibles estrategias terapéuticas del TEL, en tanto se ha planteado como una característica del trastorno las dificultades en el uso de nexos y el desarrollo de la complejidad sintáctica, dentro de lo cual los conectores son parte de esos fenómenos del discurso. A su vez, investigaciones de este tipo podrían dar cuenta del desarrollo del nivel léxico y morfosintáctico en edades más avanzadas, pues los marcadores constituyen parte del registro léxico necesario para la organización discursiva y se utilizan en la introducción de proposiciones complejas como la coordinación y la subordinación que se desarrollan durante esta etapa. Por estas razones, investigaciones como las que se proyectan permitirían hacer un seguimiento del desarrollo lingüístico tardío en el diagnóstico señalado.

Para finalizar, esta investigación confirma que el grupo TEL de 7 años presenta una estructura secuencial en sus narraciones orales al igual que sus pares con DT y que su uso de conectores tiene un nivel que no es estadísticamente diferente al grupo control. De todas formas, la cohesión y los conectores son elementos que deben seguir siendo trabajados sobre todo en niños con este diagnóstico, pues es uno de los recursos de la lengua que facilitará su progreso en su desempeño lingüístico y escolar. Sin duda, el fin último de la enseñanza-aprendizaje de estos mecanismos lingüísticos para la población con TEL es la optimización de la generación de estrategias de comunicación, teniendo en cuenta que actualmente la educación escolar cada vez es más inclusiva, en pro de un mejor desempeño discursivo, coherente y cohesivo que, por tanto, favorezca una mejor calidad de vida para los niños con este tipo de trastorno lingüístico.

\section{Agradecimientos}

Esta investigación ha sido posible gracias a la financiación del Proyecto Fondecyt Regular 1191481, "Inducción automática de taxonomías de marcadores discursivos a partir de corpus multilingües", dirigido por Rogelio Nazar. Agradecemos también a la Prof. Nina Crespo por compartir el corpus y a los revisores anónimos de la revista por sus valiosos aportes. 


\section{REFERENCIAS}

Acosta, V. González, N. y Lorenzo, C. (2011). Un análisis cualitativo de la estructura episódica, los recursos cohesivos y la diversidad léxica en la narrativa del alumnado con Trastorno Específico del Lenguaje, Revista de Psicología, 28, 143-159.

Acosta, V., Ramírez, G.M. y Hernández, S. (2017). Funciones ejecutivas y lenguaje en subtipos de niños con trastornos específicos del lenguaje. Revista de Neurología, 32(6), 355-362.

Acosta, V., Hernández, N. y Ramírez, G. (2018). Los problemas en la cohesión del discurso narrativo en alumnado con Trastorno Específico del Lenguaje (TEL), Onomázein, 39, 169-187.

Acosta, V., Ramírez, G. y Hernández, N. (2018). La producción gramatical en el discurso narrativo de alumnado con Trastorno Específico del Lenguaje (TEL). Revista signos, 51(98), 264-284.

Adam, J.M. y Lorda, C.U. (1999). Lingüistica de los textos narrativos. Barcelona, España: Editorial Ariel.

Aguado, G., Cuetos-Vega, F., Domezáin, M. y Pascual, B. (2006). Repetición de pseudopalabras en niños españoles con trastorno específico del lenguaje: marcador psicolinguiístico. Revista de Neurología, 43, 201-208.

Alfaro, P., Crespo, N. y Alvarado, C. (2016). Complejidad sintáctica en narraciones de niños con desarrollo típico, trastorno específico del lenguaje y discapacidad intelectual. Sintagma, 28, 27-41.

Álvarez, G. (1996). Conexión textual y escritura en narraciones escolares. Onomázein, 1. 11-29.

Aravena, S. (2010). El desarrollo narrativo desde la infancia a la adultez: análisis de la referencia de persona, tiempo y espacio y su relación con la estructura narrativa (tesis de doctorado). Universidad de Barcelona, España.

Asociación Americana de Psiquiatría. (2014). Manual diagnóstico y estadístico de los trastornos mentales (DSM-V). España: Editorial médica panamericana.

Blakemore, D. (2004). Relevance and Linguistic Meaning The semantic and pragmatics of Discourse Markers. England: Cambridge University Press.

Bloom, L., Lahey, M., Lifter, K. y Fiess, K. (1980). Complex sentences: Acquisition of syntactic connectives and the meaning relations they encode. Journal of Child Language, 7, 235-261. Reprinted in Bloom, L. (1991). Language development from two to three (261-288). New York: Cambridge University.

Buiza, J., Rodríguez, M. y Adrián, J. (2015). Trastorno Específico del Lenguaje: Marcadores psicolingüísticos en semántica y pragmática en niños españoles. Anales de psicología, 31(3), 879-889.

Calsamiglia, H. y Tusón, A. (1999). Las cosas del decir: Manual de análisis del discurso. España: Ariel. 
Casado Velarde, M. (1993). Introducción a la Gramática del texto de español. Madrid, España: Arco libros.

Cisternas, I. y León, N. (2018). Perfil narrativo de niños con trastorno específico del lenguaje, Revista de estudios y experiencias en educación, 17 (13), 47-68.

Coloma, J. (2013). Sintaxis compleja y discurso narrativo en escolares con Trastorno Específico del Lenguaje (TEL) (tesis doctoral). Editorial Universidad de Granada.

Coloma, J. (2014). Discurso narrativo en escolares de 1 ro básico con Trastorno Específico del Lenguaje (TEL), Revista Signos, 47 (84), 3-20.

Coloma, J., Mendoza, G. y Carballo, E. (2017). Desempeño gramatical y narrativo en niños con Trastorno Específico del Lenguaje. Círculo de Lingüística Aplicada a la Comunicación, 69, 67-90.

Crespo, N. y Figueroa, A. (2016). Los perfiles narrativos de niños con condiciones lingüísticas y cognitivas distintas. Literatura y lingüísticas, (33), 443-464.

Crespo, N., Koza, W., y Alfaro, P. (2018). Elisión y sustitución de las preposiciones en español en narraciones de niños con Trastorno específico del lenguaje. Revista de lingüística teórica y aplicada, 56(1), 161-184.

De Beaugrande, R. y Dressler, W. (1981). Introduction to text linguistics. Essex, England: Longman.

Figueroa, V., Menares, S., Naranjo, M. y Vergara, C. (2015). Mecanismos de cohesión utilizados en narraciones por un grupo de niños con TEL (tesis de pregrado). Universidad de Chile.

Gili Gaya, S. (1980). Curso superior de sintaxis española. Barcelona: Bibliograf S.A. Gutiérrez-Clellen, V.F. e Iglesias, A. (1992). Causal coherence in the oral narratives of Spanish-speaking children. Journal of Speech and hearing research, 35, 363-372.

Jackson-Maldonado, D. y Maldonado, R. (2016). El uso de conectores en niños con y sin Trastorno Específico del Lenguaje, Lingüistica Mexicana, 8 (2), 3350.

Kaderavek, J. y Sulzby, E. (2000). Narrative production by children with and without Specific Language Impairment: Oral narratives and emergent readings. Journal of speech, language and hearing research, 43, 34-49.

Lepe, N. (2018). Efectos de los gestos en la memoria de trabajo y el lenguaje oral de niños con trastorno del lenguaje (tesis doctoral). Universidad de Concepción, Chile.

López-Ornat, S. (1999). La adquisición del lenguaje. Nuevas perspectivas. Capítulo 9, en F. Cuetos \& M. de Vega (Coords): Avances en la Psicolingüística del español. Trotta, Editors: M. de Vega y F. Cuetos, pp. 469-533.

Maggiolo, M., Coloma, C. J. y Pavez, M. M. (2009). Estimulación de narraciones infantiles. Revista CEFAC, 11 (N³), 379-388. 
Mantiñán, N., Badel, M. y Fermoselle, M. (2014). Lenguaje y memoria de trabajo: implicancias en la detección e intervención del TEL. Revista Neuropsicología Latinoamericana, 6(3), 47-54.

Marinkovich, J. (1985). La adquisición de conectivos conjuntivos en español. Revista signos, 18(23), 83-95.

Martín Zorraquino, M. A. y Portolés, J. (1999). Los marcadores del discurso. En I. Bosque \& V. Demonte (Eds.) Gramática descriptiva de la lengua española, Vol. 2. Madrid: Espasa, pp. 4051-4213.

Mineduc, Ministerio de Educación, Gobierno de Chile. (2002). Decreto exento $\mathrm{n}^{\circ} 1300$ Aprueba Planes y Programas de Estudio para alumnos con trastornos específicos del lenguaje. Santiago. Disponible en: http://especial.mineduc.cl/ wp-content/uploads/sites/31/2016/08/201304231710590.DecretoN1300. pdf [Consulta: 29/6/2020].

Mineduc, Ministerio de Educación, Gobierno de Chile. (2013). Lenguaje y comunicación Programa de Estudio de Tercer Año Básico. Santiago, Chile. Disponible en: https://www.curriculumnacional.cl/614/articles-18962_programa.pdf [Consulta: 29/6/2020].

Pavez, M. M. (2003). Test exploratorio de gramática española de A. Toronto. Santiago, Chile: Ediciones UC.

Pérez, E. (1997). Cohesión y coherencia en las narraciones de niños y niñas con Trastorno Específico del Lenguaje, Revista de Logopedia, Foniatría y Audiología, 7 (2), 103-111.

R Foundation. (2020). The R project for statistical computing. Disponible en: https://www.r-project.org/ [Consulta: 29/6/2020].

Rosemberg, C., Menti, A., Stein, A., Alam, F. y Migdalek, M. (2016). Vocabulario, narración y argumentación en los primeros años de la infancia y la niñez. Una revisión de investigaciones. Revista costarricense de psicología, 35(2), 74-93.

Soriano, M. y Contreras, M. (2004). El valor de la narrativa en la caracterización de los alumnos con dificultades del lenguaje. Revista de Logopedia, Foniatría y Audiología, 24 (3), 119-125.

Soriano, M. y Contreras, M. (2012). Narraciones escritas en niños con Trastorno Específico del Lenguaje (TEL). Universitas Psychologica, 11(4), 1341-1351.

Van Dijk, T. (1978). La ciencia del texto. Barcelona, España: Paidós.

Vygotski, L. S. (1981). The genesis of higher mental functions. En J.Wersch (Ed.) The concept of activity in societ psychology. Armonk, Nueva York: Sharpe, M. E. 


\section{ANEXO}

Material inédito de Fondecyt 130420

El valiente Lilo

Había una vez un pajarito azul de alas verdes y gran corazón, llamado Lilo. Lilo vivía con sus hermanos Paca y Toto en el Bosque de Mil Colores. Su casa era un nido en la rama más alta de un gran roble. Todos los días su madre llegaba con la comida para cada uno de sus hijos.

Un día la mamá comenzó a volar, frente al nido, mostrándole cómo debían mover las alas, para que ellos la imitaran. Mañana haremos nuestra primera práctica de vuelo, les dijo. Paca y Toto cantaron alegremente, pero Lilo se quedó callado, porque sintió mucho miedo. El pajarito se preguntaba:

- ¿Y si me caigo al volar? ¿Dolerá mucho caer desde tanta altura?

Lilo pasó toda la noche preocupado y, de repente, se le ocurrió una idea: se haría el enfermo para no tener que ir a la práctica de vuelo. A la mañana siguiente, mientras sus hermanos practicaban, Lilo se volvió a acostar, se tapó los ojos con las alas y llorando se quejaba:

- Me duelen los ojos, hoy no podré practicar.

Así pasaron los días y, mientras sus hermanos volaban por todo el Bosque de Mil Colores, él solo los miraba. Su madre estaba cada vez más preocupada porque el pequeño Lilo no lograba aprender a volar. Una mañana mientras Lilo estaba escondido en el nido, vio que su hermana Paca estaba en peligro, porque una serpiente quería devorársela. Lilo se sintió muy angustiado, pues quería ayudar a su hermana, pero no había aprendido a volar por temor a caerse. Entonces, en ese momento, pensó:

- No tendré más miedo, imitaré el vuelo de mi mamá y así salvaré a Paca.

Comenzó a batir sus alas, cada vez más y más rápido, y tal como lo hacía su madre, se impulsó y, desde la rama, dio un enorme salto. Sin darse cuenta, Lilo estaba volando. Con su piquito tomó una rama y golpeó a la serpiente, la que arrancó asustada y se escondió por el bosque. Paca, al verse liberada, fue hacia su hermano y juntos volaron al nido. Lilo se sintió muy feliz de haber vencido el miedo a volar y haber salvado a su hermana. Esa noche, todos juntos celebraron la gran hazaña del pequeño pajarito azul y lo felicitaron por su valentía. 